\title{
Cryoablation of atrial fibrillation complicated by acute bacterial endocarditis
}

\author{
Romain Cassagneau • Peggy Jacon • Alix Martin • \\ Pascal Defaye
}

Received: 25 July 2012 /Accepted: 29 November 2012 /Published online: 16 March 2013

(C) Springer Science+Business Media New York 2013

Keywords Catheter ablation · Atrial fibrillation ·

Cryoablation $\cdot$ Endocarditis

\section{Introduction}

A 62-year-old man was referred for ablation of atrial fibrillation (AF). On a pre-procedural echocardiogram, left atrium and mitral valve were normal. Pulmonary vein isolation was performed, using one hexapolar catheter, 23 and $28 \mathrm{~mm}$ cryoballoons, one intracardiac echocardiography catheter, one microcircular octopolar and one standard decapolar lasso mapping catheters. Total procedure duration was $200 \mathrm{~min}$; cumulative duration of cryoapplication was $52.5 \mathrm{~min}$. No linear radiofrequency lesion was performed. He was discharged from the hospital $48 \mathrm{~h}$ later. On post-procedure day 5 , he experienced mental confusion, chills and fever. Upon readmission, he was in severe sepsis with a left central facial palsy and bilateral absence of radial pulses. Imaging studies revealed embolec infarcts, in no atrio-oesophageal fistula and, on transoesophageal echocardiogram, a $12-\mathrm{mm}$ vegetation attached to the posterior mitral leaflet (Fig. 1). Methicillinsensitive Staphylococcus aureus was grown. He was placed on a regimen of double antimicrobial therapy for 6 weeks and underwent bilateral surgical revascularization of the upper extremities, with findings of mycotic aneurysms. After 1 year of follow-up, he was in stable health and free from AF, without neurological sequelae or mitral valve regurgitation.

R. Cassagneau $\cdot$ P. Jacon $\cdot$ A. Martin $\cdot$ P. Defaye Department of Electrophysiology and Cardiac Pacing,

University Hospital of Grenoble, Grenoble, France

R. Cassagneau $(\bowtie)$

Department of Cardiology, University Hospital,

38043 Grenoble, France

e-mail: rcassagneau@chu-grenoble.fr

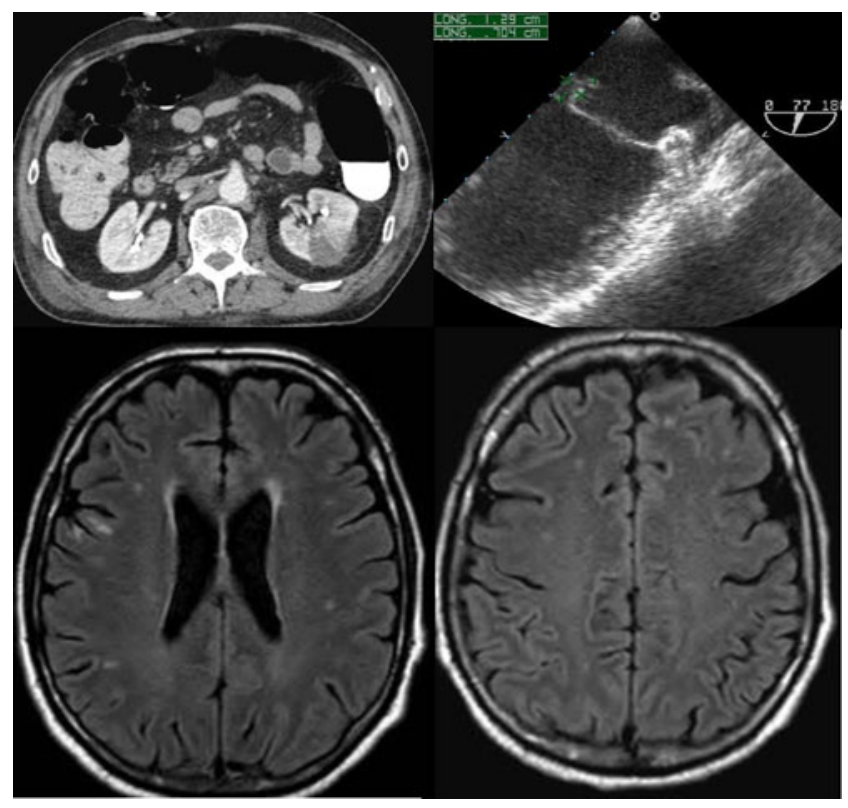

Fig. 1 Upper panels: abdominal CT scanner showing a left renal infarct; TEO reveals a vegetation attached to the posterior mitral leaflet; Inferior panels: brain MRI showing multiple and bilateral cerebral delayed enhancement signals

S. aureus may lead to a fulminating infectious process [1] and infective endocarditis has been reported after radiofrequency ablation. The unusually lengthy cryoapplication and the exchanges of multiple catheters may have a role.

Conflict of interest The authors have no conflict of interest to disclose.

\section{References}

1. Sinha, B., \& Herrmann, M. (2005). Mechanism and consequences of invasion of endothelial cells by Staphylococcus aureus. Thrombosis and Haemostasis, 94, 266-277. 